\title{
MATERNAL STRESS DURING PREGNANCY AND CORD FERRITIN CONCENTRATIONS
}

\author{
R. Armony-Sivan ${ }^{1}$, S. Aviner ${ }^{2,3}$, L. Cojocaru ${ }^{1}$, H. Babkoff ${ }^{1}$, S. Fytlovich ${ }^{3}$, D. Ben Alon ${ }^{3}$, E. Anteby ${ }^{2,3}$ \\ ${ }^{1}$ Ashkelon Academic College, Ashkelon, ${ }^{2}$ Ben Gurion University of the Negev, Beer-Sheva, ${ }^{3}$ Barzilai Medical \\ Center Campus, Ashkelon, Israel
}

Background and aims: Coe et al (2007) reported that maternal stress in monkeys during pregnancy is a risk factor for iron deficiency in infancy. The purpose of the present study was to compare cord ferritin concentration in newborns whose mothers were resident in a war zone during the first trimester of pregnancy (stress group) to those of newborns whose mothers became pregnant after the war (control group).

Methods: One-hundred forty healthy mothers with no perinatal complications were recruited for the study in the delivery room. Sixty-three mothers were resident in a war zone during the first trimester of pregnancy and 77 became pregnant after the war. Cord-blood samples were taken to assess neonate iron status. Mothers were interviewed one day after delivery. Assessments of subjective stress during pregnancy were measured by a visual analog scale.

Results: Cord Ferritin levels were lower for the stress group compared with the control group (145.71 \pm 61.9 and $169.26 \pm 85.4 ; \mathrm{p}=.06$ ). A Q-Q plot of the distribution of cord ferritin showed a clear separation between the two groups beginning with the third centile. From the fourth centile on the ferritin cord concentrations in the control group were always above that of the stress group $(\mathrm{p}<.1$ by Kolmogorov-Smirnov goodness-of-fit test). Hierarchic regression showed that subjective stress predicts cord Ferritin overall $(\beta=-.18, p<.05)$, and specifically in the stress group $(\beta=-.37, \mathrm{p}, .01)$.

Conclusion: Our findings indicate that maternal subjective long-term stress during the first trimester of pregnancy is associated with lower concentrations of cord serum Ferritin. 